\title{
Surgical Compliance and Survival Outcomes for Patients with Stage TI-2 Non-Small-Cell Lung Cancer
}

This article was published in the following Dove Press journal: Cancer Management and Research

\author{
Siben Wang ${\left(\mathbb{D}^{1}\right.}^{1, *}$ \\ Weipu $\mathrm{Mao}^{2, *}$ \\ Yi Wang ${ }^{3, *}$ \\ Xiuquan $\mathrm{Shi}^{4}$ \\ Wei Wang (D) $^{3}$ \\ Lili Dai $\mathbb{I D}^{3}$ \\ Wenping Zhang'
}

'Department of Thoracic Surgery, Heping Hospital Affiliated to Changzhi Medical College, Changzhi, Shanxi Province 046000, People's Republic of China; ${ }^{2}$ Department of Urology, Affiliated Zhongda Hospital of Southeast University, Nanjing 210009, People's Republic of China; ${ }^{3}$ Department of Respiratory Medicine, The Forth Affiliated Hospital of Anhui Medical University, Hefei, Anhui Province 230022, People's Republic of China; ${ }^{4}$ Department of General Surgery, The People's Hospital of Yingshang, Fuyang, Anhui Province 236200, People's Republic of China

*These authors contributed equally to this work
Correspondence: Wenping Zhang; Lili Dai $\mathrm{Tel} / \mathrm{Fax}+86-134035580000$;

$\mathrm{Tel} / \mathrm{Fax}+86-1862157 \mid 327$

Email CZYXYZWP2019@163.com;

277|937|4@qq.com
Introduction: Our aim was to determine the relationship between surgical compliance and survival outcomes in patients with stage T1-2 non-small-cell lung cancer (NSCLC).

Methods: Patients with T1-2 NSCLC who were diagnosed between 2004 and 2015 were identified from the SEER database. Multivariate logistic regression was used to analyse factors associated with surgical compliance. Kaplan-Meier curves and Cox regression were used to analyse the effects of surgical compliance on overall survival (OS) and cancer-specific survival (CSS).

Results: Of the 221,704 eligible T1-2 NSCLC patients, 106,668 patients recommended surgery. Among them, 99,672 (93.4\%) patients were surgical compliance group, and 6996 (6.6\%) were surgical noncompliance group. Poor surgical compliance was associated with earlier diagnosis time, old age, male, black race, unmarried status, main bronchus site, poor grade/stage, and lower household income. Patients' compliance was an independent prognostic factor for OS and CSS of T1-2 NSCLC patients. Multivariate Cox regression showed that surgical noncompliance individuals showed lower OS (hazard ratio [HR] 2.494; 95\% confidence interval [CI] $2.423-$ 2.566, $p<0.001$ ) and lower CSS (HR 2.877; 95\% CI 2.782-2.974, $p<0.001$ ) compared with surgical compliance patients. In addition, results in the non-surgical group were observed to be similar to those of the surgical noncompliance group.

Conclusion: We found that patients' compliance was an independent prognostic factor for survival in T1-2 NSCLC patients. Poor surgical compliance was associated with earlier diagnosis time, old age, male, black race, unmarried status, main bronchus site, poor grade/ stage, and lower household income.

Keywords: non-small-cell lung cancer, surgical compliance, survival outcome, SEER

\section{Introduction}

Lung cancer is the second most common malignant tumour in the United States. In 2018, the number of newly diagnosed cases of lung cancer ranked second among new malignant tumours both in men and women, and the number of deaths in malignant tumour both in male and female ranked first. ${ }^{1}$ The most common histological subtype of lung cancer is non-small-cell lung cancer (NSCLC), accounting for about $85 \%,{ }^{2}$ of which lung adenocarcinoma and lung squamous cell carcinoma are the most common subtypes. ${ }^{3}$ NSCLC is an invasive subtype with high mortality, although great progress has been made in early diagnosis and treatment, there were still many cases diagnosed as advanced and with poor prognosis. ${ }^{4}$

The treatment of NSCLC includes surgery, chemotherapy, radiotherapy and drug therapy. ${ }^{5-8}$ For patients with early NSCLC, surgical treatment is the preferred method 
Table I Characteristics for TI-2 NSCLC Patients in Our Study

\begin{tabular}{|c|c|c|c|c|}
\hline \multirow[t]{2}{*}{ Characteristic } & \multirow{2}{*}{$\begin{array}{l}\text { All Patients } \\
\text { N. (\%) }\end{array}$} & \multirow{2}{*}{$\begin{array}{l}\text { Surgical Compliance Group } \\
\text { N. (\%) }\end{array}$} & \multirow{2}{*}{$\begin{array}{l}\text { Surgical Noncompliance Group } \\
\text { N. (\%) }\end{array}$} & \multirow[t]{2}{*}{ P value } \\
\hline & & & & \\
\hline Total & 106,668 & $99,672(93.4)$ & $6996(6.6)$ & \\
\hline $\begin{array}{l}\text { Year of diagnosis } \\
2004-2007 \\
2008-2011 \\
2012-2015\end{array}$ & $\begin{array}{l}35,664(33.4) \\
36,573(34.3) \\
34,431(32.3)\end{array}$ & $\begin{array}{l}32,905(33.0) \\
34,153(34.2) \\
32,614(32.7)\end{array}$ & $\begin{array}{l}2759(39.4) \\
2420(34.6) \\
1817(26.0)\end{array}$ & $<0.001$ \\
\hline $\begin{array}{l}\text { Age at diagnosis } \\
\qquad 72 \text { years } \\
72-79 \text { years } \\
>79 \text { years }\end{array}$ & $\begin{array}{l}63,556(59.6) \\
29,800(27.9) \\
13,312(12.5)\end{array}$ & $\begin{array}{l}60,503(60.7) \\
27,754(27.8) \\
11,415(11.5)\end{array}$ & $\begin{array}{l}3053(43.6) \\
2046(29.2) \\
1897(27.1)\end{array}$ & $<0.001$ \\
\hline $\begin{array}{l}\text { Sex } \\
\qquad \text { Male } \\
\text { Female }\end{array}$ & $\begin{array}{l}51,801(48.6) \\
54,867(51.4)\end{array}$ & $\begin{array}{l}48,062(48.2) \\
51,610(51.8)\end{array}$ & $\begin{array}{l}3739(53.4) \\
3257(46.6)\end{array}$ & $<0.001$ \\
\hline $\begin{array}{l}\text { Race } \\
\text { White } \\
\text { Black } \\
\text { Others }\end{array}$ & $\begin{array}{l}90,572(84.9) \\
9291(8.7) \\
6805(6.4)\end{array}$ & $\begin{array}{l}84,979(85.3) \\
8325(8.4) \\
6368(6.4)\end{array}$ & $\begin{array}{l}5593(79.9) \\
966(13.8) \\
437(6.2)\end{array}$ & $<0.001$ \\
\hline $\begin{array}{l}\text { Marital status } \\
\text { Yes } \\
\text { No } \\
\text { Unknown }\end{array}$ & $\begin{array}{l}60,311(56.5) \\
42,275(39.6) \\
4082(3.8)\end{array}$ & $\begin{array}{l}57,263(57.5) \\
38,719(38.8) \\
3690(3.7)\end{array}$ & $\begin{array}{l}3048(43.6) \\
3556(50.8) \\
392(4.6)\end{array}$ & $<0.001$ \\
\hline $\begin{array}{l}\text { Primary site } \\
\text { Main bronchus } \\
\text { Upper lobe } \\
\text { Middle lobe } \\
\text { Lower lobe } \\
\text { Overlapping } \\
\text { Lung, NOS }\end{array}$ & $\begin{array}{l}746(0.7) \\
62,232(58.3) \\
5782(5.4) \\
35,092(32.9) \\
1208(1.1) \\
1608(1.5)\end{array}$ & $\begin{array}{l}582(0.6) \\
58,218(58.4) \\
5431(5.4) \\
32,948(33.1) \\
1163(1.2) \\
1330(1.3)\end{array}$ & $\begin{array}{l}164(2.3) \\
40 \mid 4(57.4) \\
35 \mid(5.0) \\
2144(30.6) \\
45(0.6) \\
278(4.0)\end{array}$ & $<0.001$ \\
\hline $\begin{array}{l}\text { Grade } \\
\text { Grade I } \\
\text { Grade II } \\
\text { Grade III } \\
\text { Grade IV } \\
\text { Unknown }\end{array}$ & $\begin{array}{l}15,413(14.4) \\
42,573(39.9) \\
34,619(32.5) \\
2065(1.9) \\
11,998(11.2)\end{array}$ & $\begin{array}{l}|4,99|(\mid 5.0) \\
4 \mid, 486(4 \mid .6) \\
32,854(33.0) \\
1960(2.0) \\
838 \mid(8.4)\end{array}$ & $\begin{array}{l}422(6.0) \\
1087(15.5) \\
1765(25.2) \\
105(1.5) \\
3617(51.7)\end{array}$ & $<0.001$ \\
\hline $\begin{array}{l}\text { N stage } \\
\text { No } \\
\text { NI } \\
\text { N2 } \\
\text { N3 } \\
\text { NX }\end{array}$ & $\begin{array}{l}81,200(76.1) \\
11,942(11.2) \\
11,947(11.2) \\
882(0.8) \\
697(0.7)\end{array}$ & $\begin{array}{l}77,383(77.6) \\
11,381(11.4) \\
10,085(10.1) \\
415(0.4) \\
408(0.4)\end{array}$ & $\begin{array}{l}38 \mid 7(54.6) \\
561(8.0) \\
1862(26.6) \\
467(6.7) \\
289(4.1)\end{array}$ & $<0.001$ \\
\hline $\begin{array}{l}\text { SEER stage } \\
\text { Localized } \\
\text { Regional } \\
\text { Distant }\end{array}$ & $\begin{array}{l}62,353(58.5) \\
39,085(36.6) \\
5230(4.9)\end{array}$ & $\begin{array}{l}59,362(59.6) \\
37,119(37.2) \\
3191(3.2)\end{array}$ & $\begin{array}{l}2991(42.8) \\
1966(28.1) \\
2039(29.1)\end{array}$ & $<0.001$ \\
\hline
\end{tabular}

(Continued) 
Table I (Continued).

\begin{tabular}{|l|l|l|l|l|}
\hline Characteristic & All Patients & Surgical Compliance Group & Surgical Noncompliance Group & P value \\
\cline { 2 - 5 } & N. (\%) & N. (\%) & N. (\%) \\
\hline Median household income & & & & $<0.001$ \\
Q1 & $23,337(21.9)$ & $21,814(21.9)$ & $1523(21.8)$ \\
Q2 & $27,340(25.6)$ & $25,215(25.3)$ & $2125(30.4)$ \\
Q3 & $26,154(24.5)$ & $24,298(24.4)$ & $1856(26.5)$ \\
Q4 & $29,837(28.0)$ & $28,345(28.4)$ & $1492(21.3)$ \\
\hline
\end{tabular}

Abbreviations: NSCLC, non-small-cell lung cancer; AJCC, American Joint Committee on Cancer; percentages may not total 100 because of rounding; Grade I, well differentiated; Grade II, moderately differentiated; Grade III, poorly differentiated; Grade IV, undifferentiated; median household income categorized into equally proportioned quartiles.

and the only radical treatment. ${ }^{9}$ For some patients with stage IIIB and IV metastatic solitary lesions, palliative surgical resection or surgery-based comprehensive treatment can also be given. ${ }^{10}$ Systemic chemotherapy is the first consideration for patients with advanced NSCLC. ${ }^{11}$ Although great progress has been made in the diagnosis and treatment of lung cancer in recent years, the current treatment situation of lung cancer was still not optimistic, and the overall 5-year survival rate was still low.

Many studies have analyzed the related factors affecting the survival and prognosis of NSCLC patients, such as tobacco prevalence, sex, age, TNM stage and histopathological classification. ${ }^{12,13}$ In addition, the choice of treatment regimen and patient's compliance are also prognostic factors in patients with NSCLC. Jayia ${ }^{14}$ found in a retrospective study that compliance can improve the prognosis of patients with lung cancer. In our study, we tried to identify factors related to surgical incompliance in patients with T1-2 NSCLC, so that clinicians could better intervene.

\section{Methods}

The data presented in our study were retrieved from the Surveillance Epidemiology and End Results (SEER) database, which funded by the National Cancer Institute. The SEER database covers approximately $28 \%$ of the US population and includes demographic information and cancer characteristics, such as diagnosis age, year of diagnosis, race, marital status, insurance status, income status, primary tumour location, tumour grade and stage, histological type, tumor-node-metastasis (TNM) stage, ${ }^{15}$ treatment modality and survival time. ${ }^{16}$ The SEER*Stat software (version 8.3.5; SEER 18 Regs Custom Data (with additional treatment field), Nov 2017 Sub (1973-2015 varying) database) was used in this study, we identified 223,933 T1-2 NSCLC patients between January 1, 2004, and December 31, 2015.
Exclusion criteria in our study were as follows: (a) unknown survival time $(n=238)$; (b) patients under 18 years of age $(n=30)$; (c) unknown income $(n=14) ;(d)$ unknown laterality $(n=723)$; (e) unsure whether to undergo surgery $(n=1224)$. Finally, we left 221,704 eligible patients diagnosed with T1-2 NSCLC.

Variable definition information about diagnostic age, year of diagnosis, sex, race, marital status, primary site, median household income, tumour grade, $\mathrm{N}$ stage, SEER stage and survival time can be found in the SEER database. Overall survival (OS) and cancer-specific survival (CSS) were the primary endpoints of the study.

We divide the diagnostic year into three parts: Group 1: 2004-2007, Group 2: 2008-2011, and Group 3: 2012-2015. We stratify the diagnostic age using X-tile software. The age of diagnosis was divided into three levels: $<72$ years, $72-79$ years, $>79$ years (Figure S1A-C).

For the marital status, patients are divided into "Married group", "Unmarried group" and "Unknown marital status group". Unmarried patients include "Single", "Separated", "Divorced" and "Widowed".

Grade was defined by the following codes: welldifferentiated (Grade 1); moderately differentiated (Grade II); poorly differentiated (Grade III); undifferentiated (Grade IV) and unknown grade.

For the SEER stage, limited to the lung or bronchial tree with no regional lymph node expansion or distant metastasis was defined as localized; ipsilateral regional lymph node and/ or regional extension is defined as the regional; distant was defined as metastasis to the contralateral thoracic or distant lymph nodes, malignant pericardium or pleural effusion, extension to areas such as the heart, spine, abdomen, contralateral lung, skeletal muscle and skin.

Chi-square analysis was performed to assess clinical characteristics associated with patient compliance. 


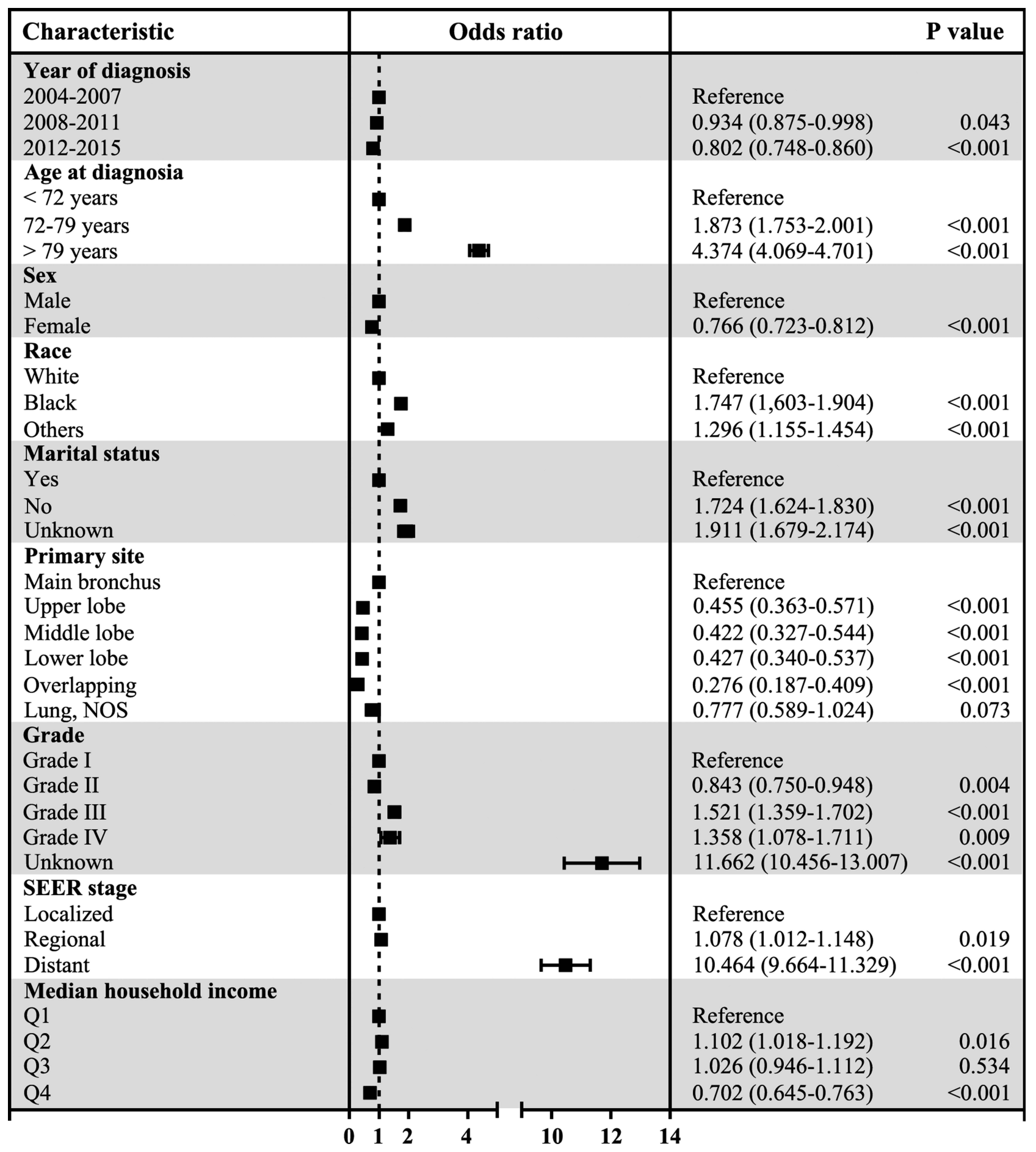

Figure I Forest plot of multivariable logistic analyses of surgical noncompliance adjusted by the year of diagnosis, age at diagnosis, sex, race, marital status, primary site, tumour grade, SEER stage and median household income. The black squares on the transverse lines represent the odds ratio (OR), and the transverse lines represent $95 \%$ $\mathrm{Cl}$. Median household income categorized into equally proportioned quartiles.

Univariate and multivariate Cox regression models were performed to estimate hazard ratios (HR) and $95 \%$ confidence interval $(\mathrm{CI})$ to analyse independent prognostic factors associated with OS and CSS in T1-2 NSCLC patients. The Kaplan-Meier curves were used to estimate OS and CSS in different groups. 1:1 propensity score matching (PSM) was to reduce the selection bias of baseline variables between groups, including age, sex, race, marital status, primary site, grade, $\mathrm{N}$ stage, SEER stage and median household income variables. The optimal diagnostic age stratification boundary 

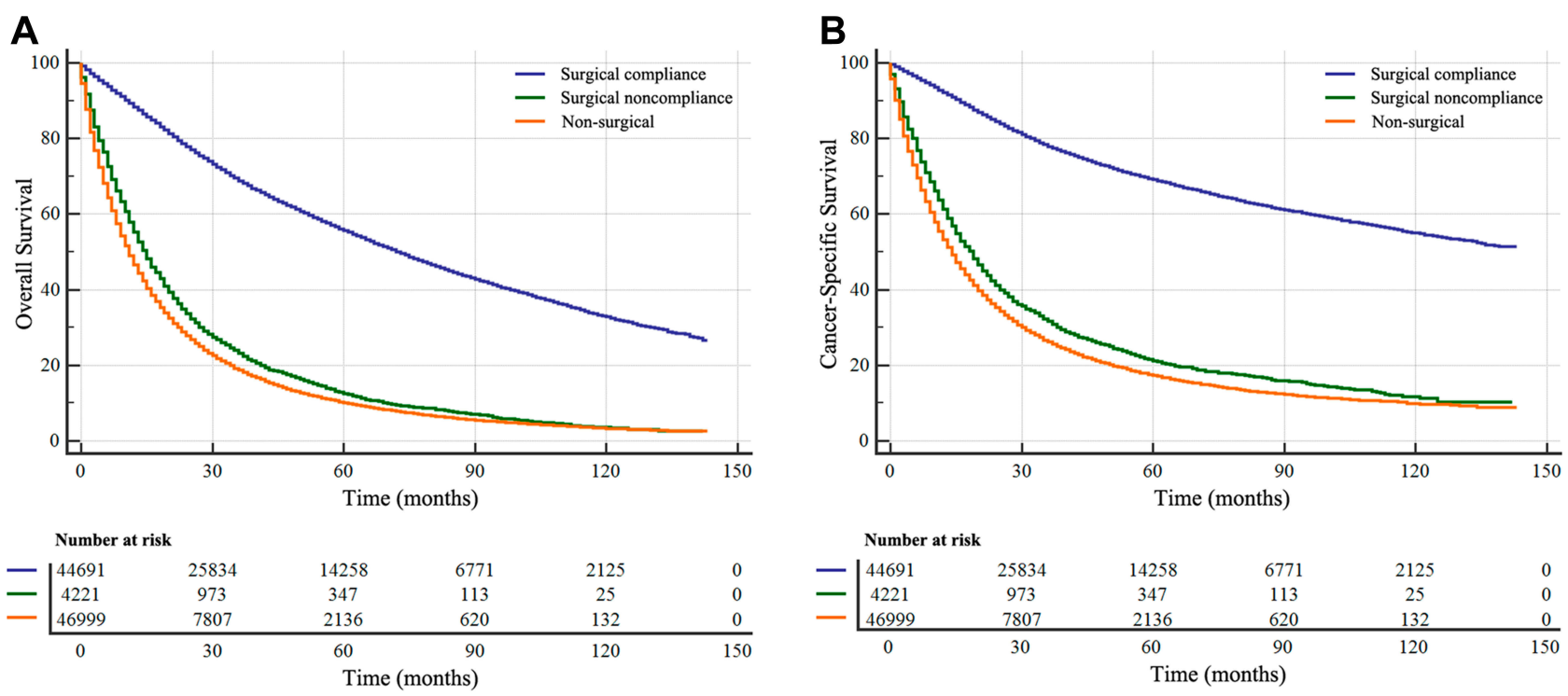

Figure 2 Kaplan-Meier survival curves according to patients' compliance (surgical compliance, surgical noncompliance, and non-surgical) in patients with TI-2 NSCLC. (A), Overall survival (OS); (B), cancer-specific survival (CSS).

was found by using X-tile software v3.6.1 (Yale University, New Haven, USA). The Social Science Software Statistics Package (version 24.0; SPSS, Chicago, USA) was used for all statistical analyses. A P value of $\leq 0.05$ was considered statistically significant.

\section{Results}

\section{Demographic and Clinical Characteristics of the TI-2 NSCLC Patients}

Our study cohort included 221,704 patients with T1-2 NSCLC, of whom 106,668 were recommended for surgery and the remaining 115,036 for non-surgical treatment. Of the patients recommended for surgical treatment, 99,672 (93.4\%) underwent surgery and $6996(6.6 \%)$ did not. Table 1 shows the correlation between surgical compliance and clinicopathological features in patients with T1-2 NSCLC. We can conclude that the non-compliance of surgery gradually decreased over time. Chi-square test showed that there were significant differences in surgical compliance among some variables, including the year of diagnosis, diagnosis age, sex, race, marital status, primary site, tumour grade, $\mathrm{N}$ stage, SEER staging and median household income (All $\mathrm{p}<0.001)$. We found that older patients (>79 years old), distant patients and low-income (Q2) patients were more likely to refuse surgery, while younger patients $(<72$ years old), localized patients and high-income (Q4) patients were more likely to receive surgical treatment.

In addition, we also analyzed the clinicopathological characteristics of surgical noncompliance group and non- surgical group used chi-square test (Table S1). We found that in addition to sex, the year of diagnosis, age at diagnosis, race, marital status, primary site, tumour grade, N stage, SEER stage and median household income were significantly different between the two groups (All $\mathrm{p}<0.001)$.

\section{Surgical Compliance-Related Factors}

Through multivariable logistic regression, we explore variables related to surgical compliance in patients with T1-2 NSCLC (Figure 1). Patients who were diagnosed recently (2012-2015: odds ratio [OR] 0.802; 95\% confidence interval [CI] 0.748-0.860, $\mathrm{p}<0.001$ ), female (OR 0.766; 95\% CI $0.723-0.812, \mathrm{p}<0.001$ ), lower lobe primary site (OR 0.427 ; $95 \%$ CI $0.340-0.537, \mathrm{p}<0.001$ ), higher income (Q4: OR 0.702; 95\% CI 0.645-0.763, p < 0.001) were more likely to follow surgical treatment.( Older $(>79$ years: OR 4.374; 95\% CI 4.069-4.701, $p<0.001)$, black race (OR 1.747; 95\% CI 1.603-1.904, $p<0.001$ ), unmarried (OR 1.724; 95\% CI 1.624-1.830, $p<0.001$ ), grade IV stage (OR 1.358; 95\% CI 1.078-1.711, $p=0.009$ ) and distant stage (OR 10.464; 95\% CI 9.664-11.329, $p<$ $0.001)$ were related to poor compliance.

\section{Identification of Prognostic Factors for OS and CSS in TI-2 NSCLC Patients}

In our study, Kaplan-Meier curve was used to analyze the influence of surgical compliance on OS and CSS of T1-2 
Table 2 Univariate and Multivariate Analysis of Overall Survival (OS) Rates

\begin{tabular}{|c|c|c|c|c|}
\hline \multirow[t]{2}{*}{ Characteristic } & \multicolumn{2}{|l|}{ Univariate Analysis } & \multicolumn{2}{|l|}{ Multivariate Analysis } \\
\hline & Hazard Ratio $(95 \% \mathrm{Cl})$ & $P$ value & Hazard Ratio $(95 \% \mathrm{Cl})$ & $P$ value \\
\hline $\begin{array}{l}\text { Age at Diagnosis } \\
\quad<72 \text { years } \\
72-79 \text { years } \\
>79 \text { years }\end{array}$ & $\begin{array}{l}\text { Reference } \\
1.239(1.224-1.254) \\
1.621 \text { (1.599-1.643) }\end{array}$ & $\begin{array}{l}<0.001 \\
<0.001\end{array}$ & $\begin{array}{l}\text { Reference } \\
1.319(1.303-1.335) \\
1.565(1.543-1.588)\end{array}$ & $\begin{array}{l}<0.001 \\
<0.001\end{array}$ \\
\hline $\begin{array}{l}\text { Sex } \\
\qquad \text { Male } \\
\text { Female }\end{array}$ & $\begin{array}{l}\text { Reference } \\
0.733(0.725-0.74 I)\end{array}$ & $<0.001$ & $\begin{array}{l}\text { Reference } \\
0.754(0.746-0.762)\end{array}$ & $<0.001$ \\
\hline $\begin{array}{l}\text { Race } \\
\text { White } \\
\text { Black } \\
\text { Others }\end{array}$ & $\begin{array}{l}\text { Reference } \\
1.128(1.109-1.146) \\
0.837(0.818-0.857)\end{array}$ & $\begin{array}{l}<0.001 \\
<0.001\end{array}$ & $\begin{array}{l}\text { Reference } \\
0.97 \mid(0.954-0.987) \\
0.802(0.784-0.82 I)\end{array}$ & $\begin{array}{l}0.001 \\
<0.001\end{array}$ \\
\hline $\begin{array}{l}\text { Marital Status } \\
\text { Yes } \\
\text { No } \\
\text { Unknown }\end{array}$ & $\begin{array}{l}\text { Reference } \\
1.167(1.154-1.179) \\
1.024(0.996-1.054)\end{array}$ & $\begin{array}{l}<0.001 \\
0.096\end{array}$ & $\begin{array}{l}\text { Reference } \\
1.170(1.157-1.183) \\
1.045(1.015-1.075)\end{array}$ & $\begin{array}{l}<0.001 \\
<0.001\end{array}$ \\
\hline $\begin{array}{l}\text { Primary Site } \\
\text { Main bronchus } \\
\text { Upper lobe } \\
\text { Middle lobe } \\
\text { Lower lobe } \\
\text { Overlapping } \\
\text { Lung, NOS }\end{array}$ & $\begin{array}{l}\text { Reference } \\
0.476(0.46 I-0.49 I) \\
0.469(0.45 I-0.487) \\
0.497(0.48 I-0.5 I 3) \\
0.498(0.468-0.530) \\
0.869(0.834-0.905)\end{array}$ & $\begin{array}{l}<0.001 \\
<0.001 \\
<0.001 \\
<0.001 \\
<0.001\end{array}$ & $\begin{array}{l}\text { Reference } \\
0.767(0.744-0.792) \\
0.796(0.766-0.827) \\
0.842(0.815-0.869) \\
0.952(0.894-1.013) \\
0.993(0.953-1.034)\end{array}$ & $\begin{array}{l}<0.001 \\
<0.001 \\
<0.001 \\
0.121 \\
0.718\end{array}$ \\
\hline $\begin{array}{l}\text { Grade } \\
\text { Grade I } \\
\text { Grade II } \\
\text { Grade III } \\
\text { Grade IV } \\
\text { Unknown }\end{array}$ & $\begin{array}{l}\text { Reference } \\
1.544 \text { (I.506-I.582) } \\
2.380(2.323-2.438) \\
2.493(2.387-2.603) \\
3.592(3.508-3.679)\end{array}$ & $\begin{array}{l}<0.001 \\
<0.001 \\
<0.001 \\
<0.001\end{array}$ & $\begin{array}{l}\text { Reference } \\
\mathrm{I} .389(\mathrm{I} .355-\mathrm{I} .424) \\
\mathrm{I} .64 \mathrm{I}(\mathrm{I} .60 \mathrm{I}-\mathrm{I} .682) \\
\mathrm{I} .755(\mathrm{I} .680-1.833) \\
\mathrm{I} .47 \mathrm{I}(\mathrm{I} .436-1.508)\end{array}$ & $\begin{array}{l}<0.001 \\
<0.001 \\
<0.001 \\
<0.001\end{array}$ \\
\hline $\begin{array}{l}\text { N Stage } \\
\text { N0 } \\
\text { NI } \\
\text { N2 } \\
\text { N3 } \\
\text { NX }\end{array}$ & $\begin{array}{l}\text { Reference } \\
1.574(1.546-1.602) \\
2.577(2.546-2.608) \\
3.091(3.031-3.152) \\
3.259(3.152-3.369)\end{array}$ & $\begin{array}{l}<0.001 \\
<0.001 \\
<0.001 \\
<0.001\end{array}$ & $\begin{array}{l}\text { Reference } \\
1.189(1.164-1.214) \\
1.288(1.268-1.309) \\
1.034(1.008-1.061) \\
1.370(1.323-1.419)\end{array}$ & $\begin{array}{l}<0.001 \\
<0.001 \\
<0.001 \\
<0.001\end{array}$ \\
\hline $\begin{array}{l}\text { SEER Stage } \\
\text { Localized } \\
\text { Regional } \\
\text { Distant }\end{array}$ & $\begin{array}{l}\text { Reference } \\
1.620(1.599-1.642) \\
4.616(4.556-4.677)\end{array}$ & $\begin{array}{l}<0.001 \\
<0.001\end{array}$ & $\begin{array}{l}\text { Reference } \\
1.446(1.427-1.466) \\
2.719(2.679-2.759)\end{array}$ & $\begin{array}{l}<0.001 \\
<0.001\end{array}$ \\
\hline $\begin{array}{l}\text { Patients' Compliance } \\
\text { Surgical compliance } \\
\text { Surgical noncompliance } \\
\text { Non-surgical }\end{array}$ & $\begin{array}{l}\text { Reference } \\
3.361(3.269-3.456) \\
4.234(4.184-4.284)\end{array}$ & $\begin{array}{l}<0.001 \\
<0.001\end{array}$ & $\begin{array}{l}\text { Reference } \\
2.494(2.423-2.566) \\
2.725(2.686-2.765)\end{array}$ & $\begin{array}{l}<0.001 \\
<0.001\end{array}$ \\
\hline $\begin{array}{l}\text { Median Household Income } \\
\text { Q1 } \\
\text { Q2 }\end{array}$ & $\begin{array}{l}\text { Reference } \\
0.869(0.857-0.882)\end{array}$ & $<0.001$ & $\begin{array}{l}\text { Reference } \\
0.916(0.903-0.930)\end{array}$ & $<0.001$ \\
\hline
\end{tabular}

(Continued) 
Table 2 (Continued).

\begin{tabular}{|c|l|l|l|l|}
\hline \multirow{2}{*}{ Characteristic } & \multicolumn{2}{|l|}{ Univariate Analysis } & \multicolumn{2}{l|}{ Multivariate Analysis } \\
\cline { 2 - 5 } & Hazard Ratio $\mathbf{( 9 5 \%} \mathbf{~ C l})$ & P value & Hazard Ratio (95\% Cl) & P value \\
\hline Q3 & $0.825(0.813-0.837)$ & $<0.001$ & $0.874(0.861-0.887)$ & $<0.001$ \\
Q4 & $0.761(0.750-0.773)$ & $<0.001$ & $0.831(0.819-0.844)$ & $<0.001$ \\
\hline
\end{tabular}

Abbreviations: OS, overall survival; AJCC, American Joint Committee on Cancer; Grade I, well differentiated; Grade II, moderately differentiated; Grade III, poorly differentiated; Grade IV, undifferentiated; median household income categorized into equally proportioned quartiles.

Table 3 Univariate and Multivariate Analysis of Cancer-Specific Survival (CSS) Rates

\begin{tabular}{|c|c|c|c|c|}
\hline \multirow[t]{2}{*}{ Characteristic } & \multicolumn{2}{|l|}{ Univariate Analysis } & \multicolumn{2}{|l|}{ Multivariate Analysis } \\
\hline & Hazard Ratio ( $95 \% \mathrm{Cl})$ & $P$ value & Hazard Ratio $(95 \% \mathrm{Cl})$ & $P$ value \\
\hline \multicolumn{5}{|l|}{ Age at Diagnosis } \\
\hline$<72$ years & Reference & & Reference & \\
\hline $72-79$ years & $1.124(1.108-1.140)$ & $<0.001$ & 1.225 (I.208-I.243) & $<0.001$ \\
\hline$>79$ years & 1.432 (I.409-I.455) & $<0.001$ & 1.425 (I.402-I.449) & $<0.001$ \\
\hline \multicolumn{5}{|l|}{ Sex } \\
\hline Male & Reference & & Reference & \\
\hline Female & $0.746(0.737-0.755)$ & $<0.001$ & $0.783(0.773-0.792)$ & $<0.001$ \\
\hline \multicolumn{5}{|l|}{ Race } \\
\hline White & Reference & & Reference & \\
\hline Black & $1.129(1.107-1.151)$ & $<0.001$ & $0.940(0.922-0.959)$ & $<0.001$ \\
\hline Others & $0.871(0.849-0.895)$ & $<0.001$ & $0.812(0.791-0.834)$ & $<0.001$ \\
\hline \multicolumn{5}{|l|}{ Marital Status } \\
\hline Yes & Reference & & Reference & \\
\hline No & I.143 (1.129-I.157) & $<0.001$ & $1.147(1.133-1.162)$ & $<0.001$ \\
\hline Unknown & $0.969(0.937-1.002)$ & 0.066 & $0.996(0.963-1.030)$ & 0.810 \\
\hline \multicolumn{5}{|l|}{ Primary Site } \\
\hline Main bronchus & Reference & & Reference & \\
\hline Upper lobe & $0.431(0.417-0.446)$ & $<0.001$ & $0.743(0.718-0.769)$ & $<0.001$ \\
\hline Middle lobe & $0.431(0.413-0.450)$ & $<0.001$ & $0.777(0.744-0.8 \mathrm{II})$ & $<0.001$ \\
\hline Lower lobe & $0.449(0.433-0.465)$ & $<0.001$ & $0.824(0.795-0.853)$ & $<0.001$ \\
\hline Overlapping & $0.491(0.458-0.526)$ & $<0.001$ & I.0II (0.943-I.084) & 0.701 \\
\hline Lung, NOS & $0.840(0.804-0.879)$ & $<0.001$ & $0.98 \mid(0.938-1.026)$ & 0.422 \\
\hline \multicolumn{5}{|l|}{ Grade } \\
\hline Grade I & Reference & & Reference & \\
\hline Grade II & $1.692(1.640-1.746)$ & $<0.001$ & I.45। (I.406-I.497) & $<0.001$ \\
\hline Grade III & $2.879(2.793-2.967)$ & $<0.001$ & $1.780(1.726-1.836)$ & $<0.001$ \\
\hline Grade IV & $3.076(2.922-3.239)$ & $<0.001$ & $1.938(1.840-2.04 I)$ & $<0.001$ \\
\hline Unknown & $4.456(4.324-4.591)$ & $<0.001$ & $1.565(1.517-1.614)$ & $<0.001$ \\
\hline \multicolumn{5}{|l|}{ N Stage } \\
\hline No & Reference & & Reference & \\
\hline NI & $1.900(1.861-1.939)$ & $<0.001$ & $1.265(1.236-1.295)$ & $<0.001$ \\
\hline N2 & $3.262(3.217-3.308)$ & $<0.001$ & $1.386(1.36 \mathrm{I}-1.4 \mathrm{II})$ & $<0.001$ \\
\hline N3 & 3.991 (3.907-4.078) & $<0.001$ & I.I23 (I.093-I.I55) & $<0.001$ \\
\hline NX & $3.905(3.762-4.054)$ & $<0.001$ & $1.450(1.393-1.508)$ & $<0.001$ \\
\hline
\end{tabular}

(Continued) 
Table 3 (Continued).

\begin{tabular}{|c|c|c|c|c|}
\hline \multirow[t]{2}{*}{ Characteristic } & \multicolumn{2}{|l|}{ Univariate Analysis } & \multicolumn{2}{|l|}{ Multivariate Analysis } \\
\hline & Hazard Ratio $(95 \% \mathrm{Cl})$ & $P$ value & Hazard Ratio $(95 \% \mathrm{Cl})$ & $P$ value \\
\hline \multicolumn{5}{|l|}{ SEER Stage } \\
\hline Localized & Reference & & Reference & \\
\hline Regional & $2.060(2.027-2.094)$ & $<0.001$ & 1.797 (1.768-1.827) & $<0.001$ \\
\hline Distant & $6.507(6.406-6.610)$ & $<0.001$ & $3.569(3.507-3.631)$ & $<0.001$ \\
\hline \multicolumn{5}{|l|}{ Patients' Compliance } \\
\hline Surgical compliance & Reference & & Reference & \\
\hline Surgical noncompliance & $4.014(3.886-4.145)$ & $<0.001$ & $2.877(2.782-2.974)$ & $<0.001$ \\
\hline Non-surgical & $5.226(5.152-5.302)$ & $<0.001$ & $3.086(3.033-3.140)$ & $<0.001$ \\
\hline \multicolumn{5}{|l|}{ Median Household Income } \\
\hline QI & Reference & & Reference & \\
\hline Q2 & $0.876(0.86 I-0.890)$ & $<0.001$ & $0.933(0.918-0.949)$ & $<0.001$ \\
\hline Q3 & $0.809(0.795-0.823)$ & $<0.001$ & $0.867(0.853-0.882)$ & $<0.001$ \\
\hline Q4 & $0.753(0.740-0.765)$ & $<0.001$ & $0.83 \mid(0.817-0.845)$ & $<0.001$ \\
\hline
\end{tabular}

Abbreviations: CSS, cancer-specific survival; AJCC, American Joint Committee on Cancer; Grade I, well differentiated; Grade II, moderately differentiated; Grade III, poorly differentiated; Grade IV, undifferentiated; median household income categorized into equally proportioned quartiles.

NSCLC patients (Figure 2). We can find that patients with surgical compliance survived longer than patients with poor compliance and the non-surgical groups. Univariate and multivariate Cox regression were used to analyze the factors associated with OS and CSS in patients with T1-2 NSCLC. As shown in Tables 2 and 3, age at diagnosis, sex, race, marital status, primary site, tumour grade, $\mathrm{N}$ stage, SEER stage, patients' compliance and median household income were factors that affect the OS and CSS in patients with T1-2 NSCLC. Multivariate Cox regression showed surgical noncompliance (vs surgical compliance; HR $=2.494,95 \%$ CI 2.423-2.566, $p<$ 0.001 ), non-surgical (vs surgical compliance; $\mathrm{HR}=2.725$, 95\% CI 2.686-2.765, $p<0.001$ ) were associated with OS (Figure 3). Similarly, in terms of CSS, multivariate Cox regression analysis also indicated patients' compliance was an independent prognostic factor for T1-2 NSCLC patients (surgical noncompliance vs surgical compliance; $\mathrm{HR}=2.877,95 \% \mathrm{CI}$ 2.782-2.974, $p<0.001$; non-surgical vs surgical compliance; $\mathrm{HR}=3.086,95 \%$ CI 3.033-3.140, $p<0.001$ ) (Figure S2). In addition, poor surgical compliance patients and non-surgical patients have a similarly poor OS and CSS.

\section{Subgroup Analysis for Evaluating the Effect of Marital Status on OS and CSS Based on SEER Stage and Grade}

Multivariate Cox regression analysis showed that diagnostic age and household income were closely related to the survival of patients. Based on age at diagnosis and household income, we further discussed the differences between surgical compliance and prognosis among the subgroup of T1-2 NSCLC patients (Table 4). We found that for OS and CSS, surgical compliance remained an independent prognostic factor for three age subgroups and four income subgroups (Figures 4 and 5). In each subgroup, the survival rate of patients in the surgical compliance group was still significantly higher than in the other two groups, and the survival of patients in the surgical noncompliance group was slightly higher than that in the non-surgical group.

\section{Discussion}

Our study first used a large, population-based database to explore the impact of surgical compliance on T1-2 NSCLC patients, and found that surgical compliance was an independent prognostic factor for OS and CSS in T1-2 NSCLC patients. Our study showed that poor surgical compliance was associated with earlier diagnosis time, old age, male, black race, unmarried status, main bronchus site, poor grade/stage and lower household income. As expected, survival analysis showed that the prognosis of the surgical noncompliance group was significantly worse than that of the surgical compliance group. We also analyzed the survival of the patients in the non-surgical group and found that the survival of the patients in the surgical noncompliance group was similar to that in the nonsurgical group. Although the proportion of patients with surgical noncompliance was decreasing by years, the 


\begin{tabular}{|c|c|c|c|}
\hline Characteristic & Hazard Ratio & & $P$ value \\
\hline $\begin{array}{l}\text { Age at diagnosis } \\
<72 \text { years } \\
72-79 \text { years } \\
>79 \text { years }\end{array}$ & $\begin{array}{ll}1 & \\
\vdots & \end{array}$ & $\begin{array}{l}\text { Reference } \\
1.319(1.303-1.335) \\
1.565(1.543-1.588)\end{array}$ & $\begin{array}{l}<0.001 \\
<0.001\end{array}$ \\
\hline $\begin{array}{l}\text { Sex } \\
\text { Male } \\
\text { Female }\end{array}$ & : & $\begin{array}{l}\text { Reference } \\
0.754(0.746-0.762)\end{array}$ & $<0.001$ \\
\hline $\begin{array}{l}\text { Race } \\
\text { White } \\
\text { Black } \\
\text { Others }\end{array}$ & 4 & $\begin{array}{l}\text { Reference } \\
0.971(0.954-0.987) \\
0.802(0.784-0.821)\end{array}$ & $\begin{array}{r}0.001 \\
<0.001\end{array}$ \\
\hline $\begin{array}{l}\text { Marital status } \\
\text { Yes } \\
\text { No } \\
\text { Unknown }\end{array}$ & 1 & $\begin{array}{l}\text { Reference } \\
1.170(1.157-1.183) \\
1.045(1.015-1.075)\end{array}$ & $\begin{array}{l}<0.001 \\
<0.001\end{array}$ \\
\hline $\begin{array}{l}\text { Primary site } \\
\text { Main bronchus } \\
\text { Upper lobe } \\
\text { Middle lobe } \\
\text { Lower lobe } \\
\text { Overlapping } \\
\text { Lung, NOS }\end{array}$ & $\begin{array}{r}1 \\
\vdots \\
\mathbf{0} \\
\mathbf{0} \\
\mathbf{0} \\
\vdots \\
\mathbf{4}\end{array}$ & $\begin{array}{l}\text { Reference } \\
0.767(0.744-0.792) \\
0.796(0.766-0.827) \\
0.842(0.815-0.869) \\
0.952(0.894-1.013) \\
0.993(0.953-1.034)\end{array}$ & $\begin{array}{r}<0.001 \\
<0.001 \\
<0.001 \\
0.121 \\
0.718\end{array}$ \\
\hline $\begin{array}{l}\text { Grade } \\
\text { Grade I } \\
\text { Grade II } \\
\text { Grade III } \\
\text { Grade IV } \\
\text { Unknown }\end{array}$ & $\square$ & $\begin{array}{l}\text { Reference } \\
1.389(1.355-1.424) \\
1.641(1.601-1.682) \\
1.755(1.680-1.833) \\
1.471(1.436-1.508)\end{array}$ & $\begin{array}{l}<0.001 \\
<0.001 \\
<0.001 \\
<0.001\end{array}$ \\
\hline $\begin{array}{l}\text { SEER stage } \\
\text { Localized } \\
\text { Regional } \\
\text { Distant }\end{array}$ & $\begin{array}{c}1 \\
\vdots \\
\vdots \\
1 \\
1\end{array}$ & $\begin{array}{l}\text { Reference } \\
1.446(1.427-1.466) \\
2.719(2.679-2.759)\end{array}$ & $\begin{array}{l}<0.001 \\
<0.001\end{array}$ \\
\hline $\begin{array}{l}\text { Patients' compliance } \\
\text { Surgical compliance } \\
\text { Surgical noncompliance } \\
\text { Non-surgical }\end{array}$ & ש & $\begin{array}{l}\text { Reference } \\
2.494(2.423-2.566) \\
2.725(2.686-2.765)\end{array}$ & $\begin{array}{l}<0.001 \\
<0.001\end{array}$ \\
\hline $\begin{array}{l}\text { Median household income } \\
\text { Q1 } \\
\text { Q2 } \\
\text { Q3 } \\
\text { Q4 }\end{array}$ & $\begin{array}{r}\vdots \\
\mathbf{a} \\
\mathbf{a} \\
\mathbf{a}\end{array}$ & $\begin{array}{l}\text { Reference } \\
0.916(0.903-0.930) \\
0.874(0.861-0.887) \\
0.831(0.819-0.844)\end{array}$ & $\begin{array}{l}<0.001 \\
<0.001 \\
<0.001\end{array}$ \\
\hline
\end{tabular}

Figure 3 Forest plot of multivariable Cox analyses of overall survival (OS) adjusted by the year of diagnosis, age at diagnosis, sex, race, marital status, primary site, tumour grade, SEER stage, patients' compliance and median household income. The black squares on the transverse lines represent the hazard ratio (HR), and the transverse lines represent $95 \% \mathrm{Cl}$. Median household income categorized into equally proportioned quartiles.

number of non-surgical patients was still increasing, and the health status of these patients shall not be ignored.

Surgical compliance has a great impact on the survival of cancer patients. $\mathrm{Liu}^{17}$ found that there was a correlation between surgical compliance and the prognosis of gastric cancer, and the survival rate of gastric cancer patients with poor surgical compliance was lower. Adesunkanmi ${ }^{18}$ surveyed 212 Nigerian breast cancer patients and found that 
Table 4 Subgroup Analyses Stratified by Diagnosis Age and Household Income for Overall Survival (OS) and Cancer-Specific Survival (CSS)

\begin{tabular}{|c|c|c|c|c|}
\hline \multirow[t]{2}{*}{ Characteristic } & \multicolumn{2}{|l|}{ os } & \multicolumn{2}{|l|}{ CSS } \\
\hline & Hazard Ratio $(95 \% \mathrm{Cl})$ & $P$ value & Hazard Ratio (95\% Cl) & $P$ value \\
\hline $\begin{array}{l}<72 \text { Years } \\
\text { Surgical compliance } \\
\text { Surgical noncompliance } \\
\text { Non-surgical }\end{array}$ & $\begin{array}{l}\text { Reference } \\
2.708(2.593-2.828) \\
2.929(2.869-2.991)\end{array}$ & $\begin{array}{l}<0.001 \\
<0.001\end{array}$ & $\begin{array}{l}\text { Reference } \\
2.962(2.82 \mathrm{I}-3.1 \mathrm{II}) \\
3.157(3.082-2.235)\end{array}$ & $\begin{array}{l}<0.001 \\
<0.001\end{array}$ \\
\hline $\begin{array}{l}\text { 72-79 Years } \\
\text { Surgical compliance } \\
\text { Surgical noncompliance } \\
\text { Non-surgical }\end{array}$ & $\begin{array}{l}\text { Reference } \\
2.449(2.323-2.58 I) \\
2.634(2.566-2.705)\end{array}$ & $\begin{array}{l}<0.001 \\
<0.001\end{array}$ & $\begin{array}{l}\text { Reference } \\
2.904(2.731-3.088) \\
3.090(2.992-3.192)\end{array}$ & $\begin{array}{l}<0.001 \\
<0.001\end{array}$ \\
\hline $\begin{array}{l}>79 \text { Years } \\
\text { Surgical compliance } \\
\text { Surgical noncompliance } \\
\text { Non-surgical }\end{array}$ & $\begin{array}{l}\text { Reference } \\
2.123(2.004-2.249) \\
2.362(2.286-2.440)\end{array}$ & $\begin{array}{l}<0.001 \\
<0.001\end{array}$ & $\begin{array}{l}\text { Reference } \\
2.629(2.453-2.817) \\
2.854(2.738-2.975)\end{array}$ & $\begin{array}{l}<0.001 \\
<0.001\end{array}$ \\
\hline $\begin{array}{l}\text { QI } \\
\text { Surgical compliance } \\
\text { Surgical noncompliance } \\
\text { Non-surgical }\end{array}$ & $\begin{array}{l}\text { Reference } \\
2.496(2.348-2.652) \\
2.638(2.564-2.713)\end{array}$ & $\begin{array}{l}<0.001 \\
<0.001\end{array}$ & $\begin{array}{l}\text { Reference } \\
2.914(2.717-3.126) \\
3.046(2.944-3.151)\end{array}$ & $\begin{array}{l}<0.001 \\
<0.001\end{array}$ \\
\hline $\begin{array}{l}\text { Q2 } \\
\text { Surgical compliance } \\
\text { Surgical noncompliance } \\
\text { Non-surgical }\end{array}$ & $\begin{array}{l}\text { Reference } \\
2.517(2.389-2.653) \\
2.776(2.699-2.856)\end{array}$ & $\begin{array}{l}<0.001 \\
<0.001\end{array}$ & $\begin{array}{l}\text { Reference } \\
2.895(2.726-3.075) \\
3.162(3.057-3.271)\end{array}$ & $\begin{array}{l}<0.001 \\
<0.001\end{array}$ \\
\hline $\begin{array}{l}\text { Q3 } \\
\text { Surgical compliance } \\
\text { Surgical noncompliance } \\
\text { Non-surgical }\end{array}$ & $\begin{array}{l}\text { Reference } \\
2.464(2.327-2.609) \\
2.688(2.608-2.77 I)\end{array}$ & $\begin{array}{l}<0.001 \\
<0.001\end{array}$ & $\begin{array}{l}\text { Reference } \\
2.842(2.659-3.038) \\
3.020(2.912-3.133)\end{array}$ & $\begin{array}{l}<0.001 \\
<0.001\end{array}$ \\
\hline $\begin{array}{l}\text { Q4 } \\
\text { Surgical compliance } \\
\text { Surgical noncompliance } \\
\text { Non-surgical }\end{array}$ & $\begin{array}{l}\text { Reference } \\
2.497(2.346-2.657) \\
2.807(2.725-2.891)\end{array}$ & $\begin{array}{l}<0.001 \\
<0.001\end{array}$ & $\begin{array}{l}\text { Reference } \\
2.872(2.672-3.086) \\
3.108(2.999-3.220)\end{array}$ & $\begin{array}{l}<0.001 \\
<0.001\end{array}$ \\
\hline
\end{tabular}

Abbreviations: OS, overall survival; CSS, cancer-specific survival; median household income categorized into equally proportioned quartiles.

compliance was associated with prognosis, and most of the patients died or lost follow-up within 1 year of diagnosis. Adham $^{19}$ found that survival was associated with treatment compliance in patients with nasopharyngeal carcinoma in Indonesia.

In our study, logistic regression showed that age was associated with surgical compliance. The risk of death increased with age, and surgical compliance remained an independent risk factor for OS and CSS in T1-2 NSCLC patients after diagnosis age stratification. This may be due to the poor physical fitness of elderly patients, suffering from a variety of complications before surgery. This may lead to more conservative treatments and poor compliance in older patients. ${ }^{20}$ Surgical treatment of lung cancer in elderly patients is associated with many complications. ${ }^{21}$ However, some studies have shown that there was no significant difference in postoperative complications and 5 -year survival rates between the old group ( $\geq 75$ years old) and the young group ( $<75$ years old). ${ }^{22}$

Household income was also associated with patient compliance. First of all, the income level was an important predictor of survival time in patients with lung cancer. ${ }^{23}$ One study suggests that socioeconomic barriers can pose a major challenge to the success of lung cancer screening programs in safety net hospitals, and income levels can affect compliance with screening follow-up. ${ }^{24}$ In addition, the level of income 
A
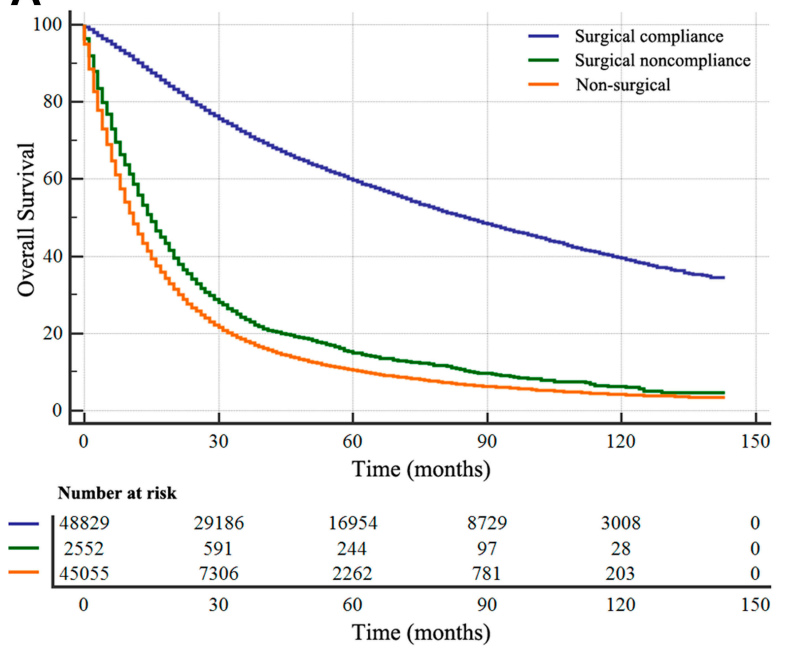

C
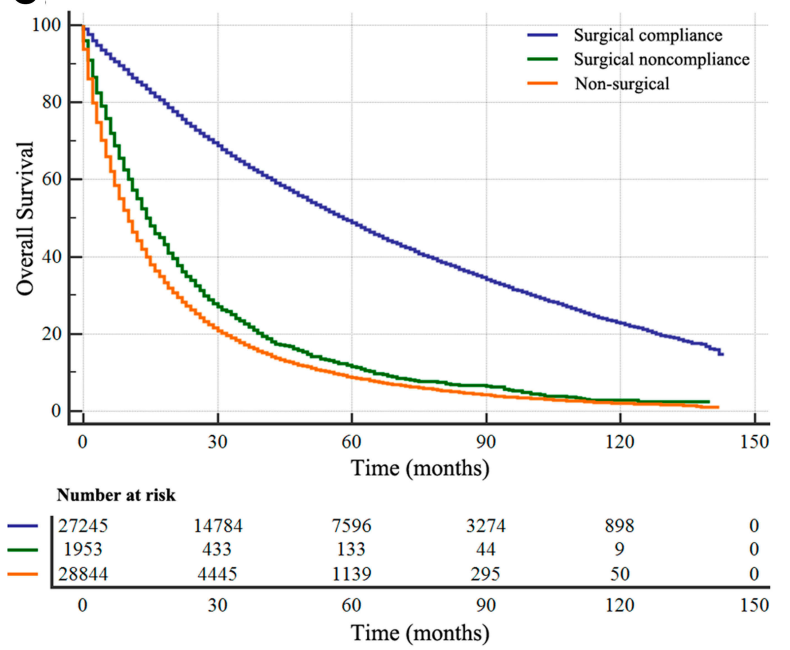

E
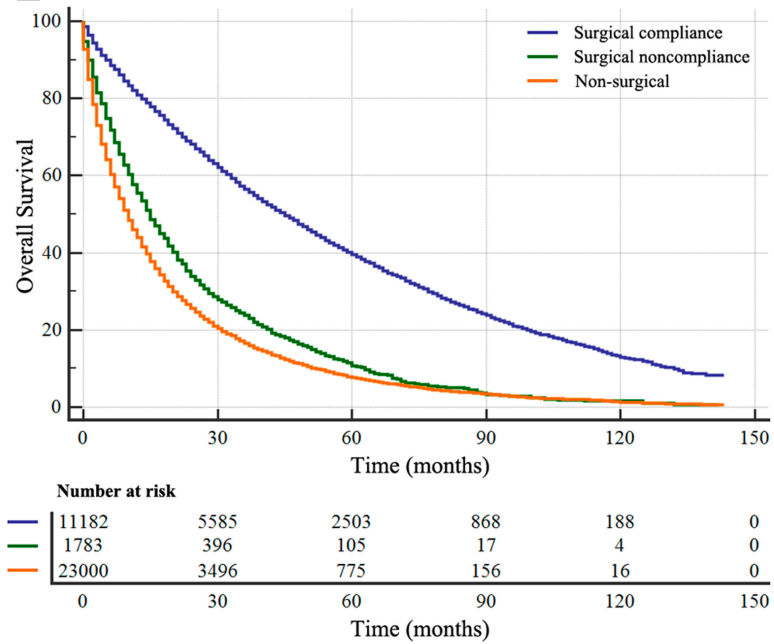

B
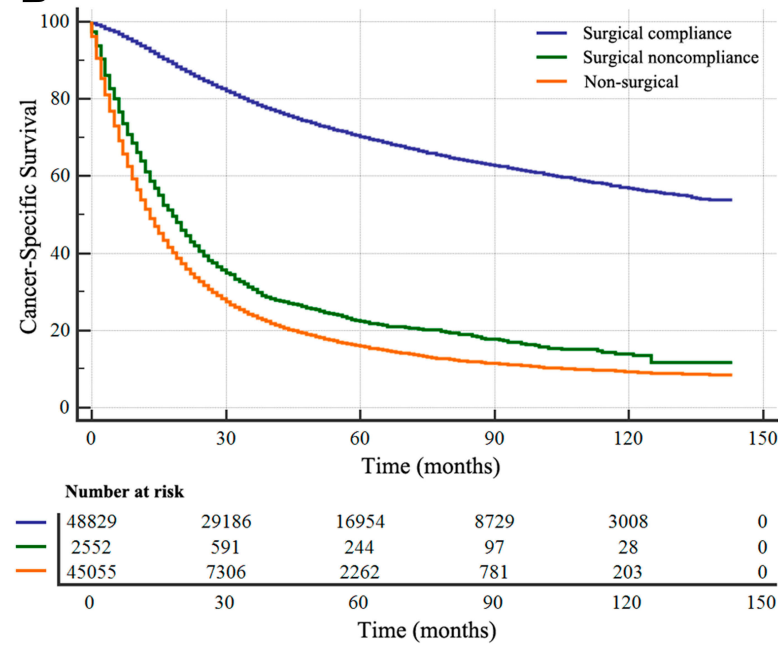

D
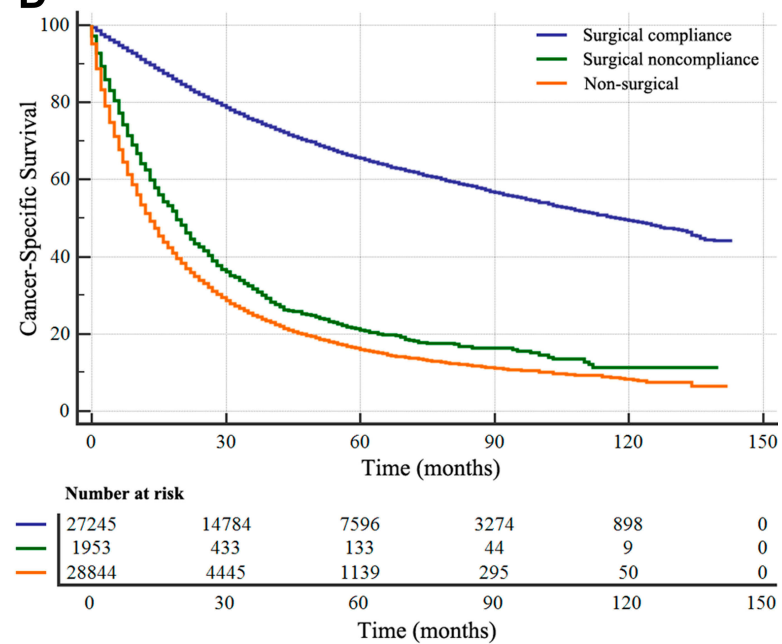

$\mathbf{F}$
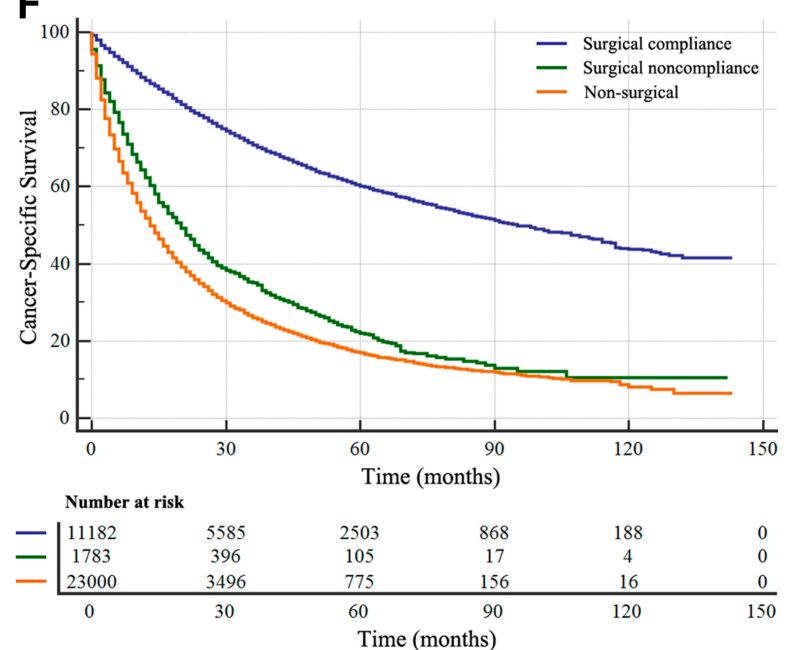

Figure 4 Overall survival (OS) and cancer-specific survival (CSS) curves of TI-2 NSCLC patients according to different diagnosis age. (A and B), OS and CSS in under 72 years patients. (C and $\mathbf{D}$ ), OS and CSS in 72-79 years patients. (E and $\mathbf{F}$ ), OS and CSS in over 79 years patients. 
A
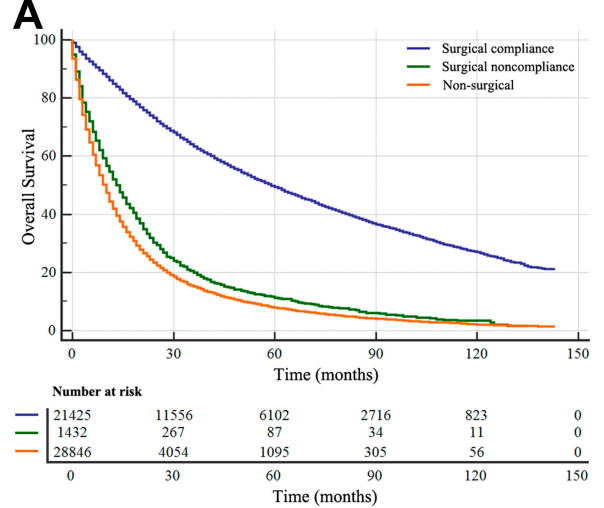

C
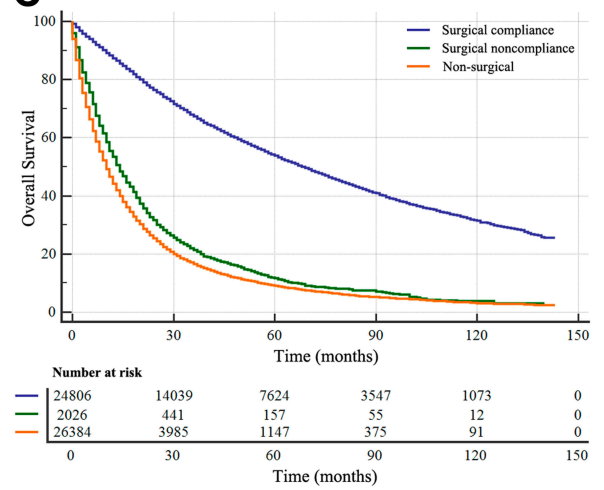

E
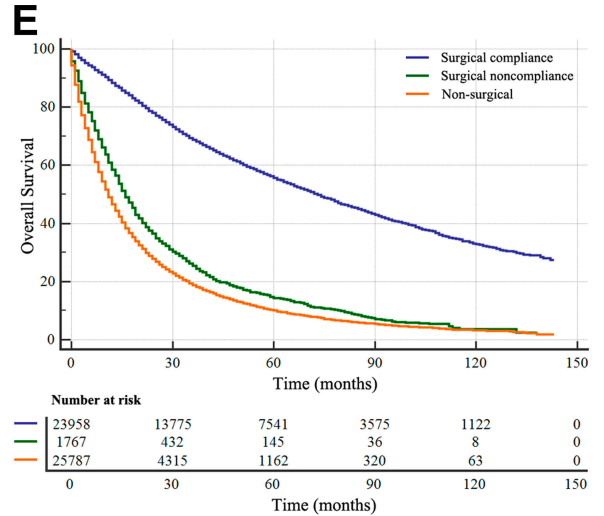

G
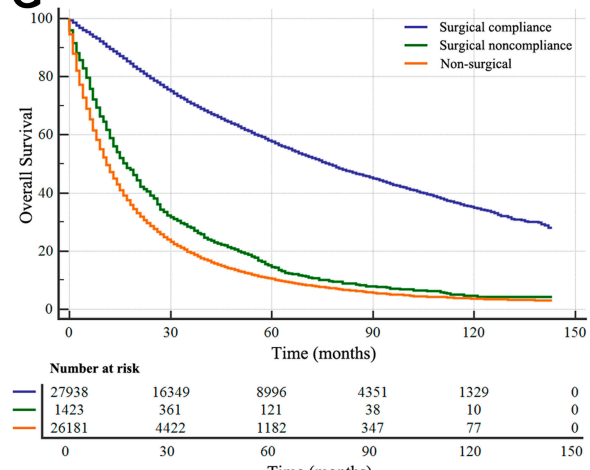

B
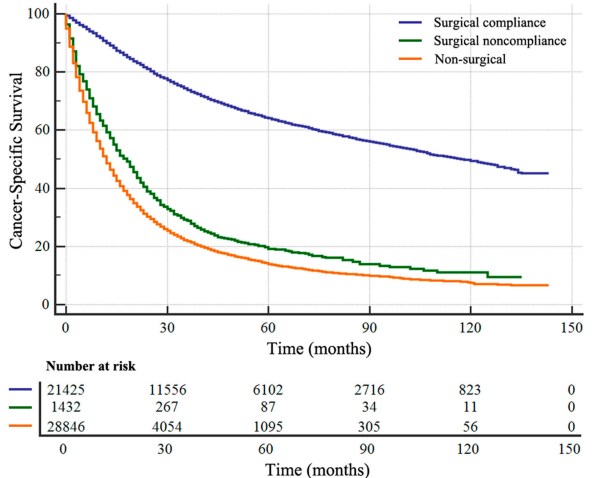

D
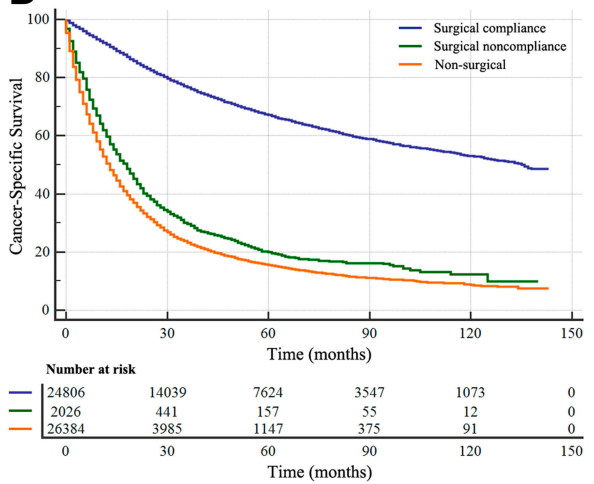

F
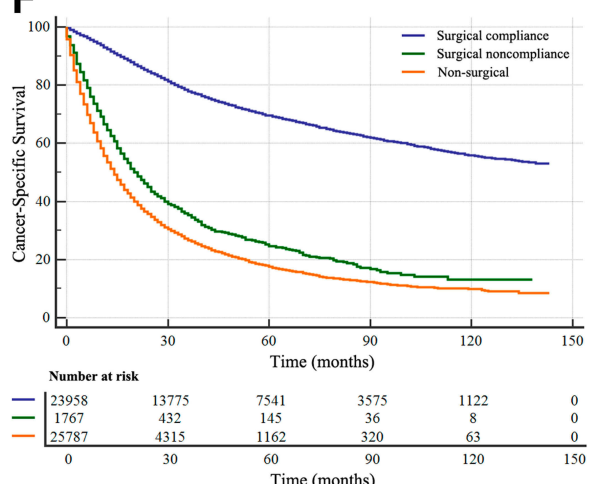

H

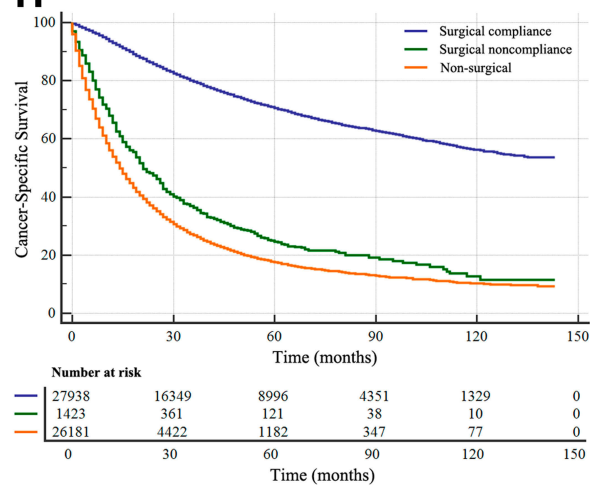

Figure 5 Overall survival (OS) and cancer-specific survival (CSS) curves of TI-2 NSCLC patients according to different household income. (A and B), OS and CSS in QI income patients. (C and D), OS and CSS in Q2 income patients. (E and F), OS and CSS in Q3 income patients. (G and $\mathbf{H})$, OS and CSS in Q4 income patients. 
will affect the use of chemotherapy in patients with II-IV NSCLC. ${ }^{25}$ Good financial condition is the guarantee of active treatment, and patients with higher household income may be more likely to receive treatment and prolong their survival time. ${ }^{26}$ Home-based interventions can achieve the highest patient compliance and improve patient's physical health or clinical symptoms..$^{27,28}$

Many studies have shown that marital status was an independent prognostic factor for the survival of a variety of cancers, including breast cancer, penis cancer, gastric cancer and colorectal cancer, and unmarried patients have a higher risk of death. ${ }^{29-32}$ Our study found that marital status was an influential factor in surgical compliance, and married patients were more likely to receive surgical treatment. We speculate that the better prognosis of married patients may be partly due to good surgical compliance.

We grouped surgical noncompliance patients and nonsurgical patients into a group. After 1:1 PSM with surgical compliance patients, a total of 99,096 patients were included in the study. We performed Kaplan-Meier curve analysis of all patients and found that surgical treatment alone can obtain the best median OS and CSS in surgical compliance patients (Figure S3A, B); for patients who have not received surgery or surgical noncompliance, radiotherapy can obtain the best median OS and CSS (Figure S3C, D).

Additionally, we also found that tumour grade and stage were also closely related to surgical noncompliance in patients with T1-2 NSCLC. This may be because patients with advanced stage of the tumour have distant metastasis or more complications at the time of diagnosis, the therapeutic effect was poor, and the patient may be less likely to undergo surgery, resulting in poor compliance with the operation. Moreover, the type of insurance was also an independent factor affecting lung cancer patient survival. Tantraworasin ${ }^{33}$ studied 102,733 lung cancer patients between 2007 and 2013 and found that Asian patients with uninsured or Medicaid-covered were less likely to receive surgery, resulting in a shorter OS.

As far as we know, this is the first SEER database-based study to focus on the surgical compliance of T1-2 NSCLC patients, but there are limitations to be recognized in this study. First, this study is a retrospective study with obvious limitations. Second, the lack of information on the physical condition and complications of patients, both of which are prognostic factors for surgical compliance. In addition, it was unclear whether the surgical noncompliance group patients used other treatments, such as chemotherapy, radiotherapy or targeted therapy.

\section{Conclusions}

In our study, we found that patients' compliance was an independent prognostic factor for survival in T1-2 NSCLC patients. Good surgical compliance had better survival, while poor surgical compliance and non-surgical patients have similarly poor OS and CSS. Poor surgical compliance was associated with earlier diagnosis time, old age, male, black race, unmarried status, main bronchus site, poor grade/stage, and lower household income.

\section{Abbreviations}

NSCLC, non-small-cell lung cancer; OS, overall survival; CSS, cancer-specific survival; SEER, Surveillance, Epidemiology, and End Results; HR, hazard ratio; CI, confidence interval; OR, odds ratio.

\section{Ethical Approval}

This study was approved by the Ethics Committee of the Heping Hospital of Changzhi Medical College (Changzhi, China) and with the 1964 Declaration of Helsinki and its later amendments or comparable ethical standards. Based on SEER website https://www.cancer.gov/policies/accessibility, the National Cancer Institute (NCI) provides access to all individuals seeking information on http://www.cancer.gov, including individuals who are disabled, and we can access data from SEER for free.

\section{Disclosure}

The authors declare there are no potential conflicts of interest related to this study.

\section{References}

1. Siegel RL, Miller KD, Jemal A. Cancer statistics, 2018. CA Cancer J Clin. 2018;68(1):7-30. doi:10.3322/caac.21442

2. Wang S, Cang S, Liu D. Third-generation inhibitors targeting EGFR T790M mutation in advanced non-small cell lung cancer. $J$ Hematol Oncol. 2016;9(1):34. doi:10.1186/s13045-016-0268-z

3. Misra P, Singh S. Role of cytokines in combinatorial immunotherapeutics of non-small cell lung cancer through systems perspective. Cancer Med. 2019;8(5):1976-1995. doi:10.1002/cam4.2112

4. Liu R, Chen Z, Yi X, et al. 9za plays cytotoxic and proapoptotic roles and induces cytoprotective autophagy through the PDK1/Akt/mTOR axis in non-small-cell lung cancer. $J$ Cell Physiol. 2019;234 (11):20728-41.

5. Ettinger DS, Akerley W, Bepler G, et al. Non-small cell lung cancer. J Natl Compr Canc Netw. 2010;8(7):740-801. doi:10.6004/jnccn. 2010.0056

6. Ohe Y, Ohashi Y, Kubota K, et al. Randomized Phase III study of cisplatin plus irinotecan versus carboplatin plus paclitaxel, cisplatin plus gemcitabine, and cisplatin plus vinorelbine for advanced non-small-cell lung cancer: four-Arm Cooperative Study in Japan. Ann Oncol. 2007;18(2):317-323. doi:10.1093/annonc/mdl377 
7. De Ruysscher D, Pijls-Johannesma M, Bentzen SM, et al. Time between the first day of chemotherapy and the last day of chest radiation is the most important predictor of survival in limited-disease small-cell lung cancer. J Clin Oncol. 2006;24(7):1057-1063. doi:10.1200/ JCO.2005.02.9793

8. Langer CJ, Manola J, Bernardo P, et al. Cisplatin-based therapy for elderly patients with advanced non-small-cell lung cancer: implications of Eastern Cooperative Oncology Group 5592, a randomized trial. J Natl Cancer Inst. 2002;94(3):173-181. doi:10.1093/jnci/94.3.173

9. Scagliotti G. Consensus development conference on the medical treatment of non-small cell lung cancer: treatment of the early stages. Lung Cancer. 2002;38(Suppl 3):S23-29. doi:10.1016/S01695002(02)00262-3

10. Kris MG, Tonato M. Proceedings of the European consensus conference on medical treatment of non-small cell lung cancer. Lung Cancer. 2002;38(Suppl 3):S1-85. doi:10.1016/S0169-5002(02)00166-6

11. Sakai H, Yoneda S, Kobayashi K, et al. Phase II study of bi-weekly docetaxel and carboplatin with concurrent thoracic radiation therapy followed by consolidation chemotherapy with docetaxel plus carboplatin for stage III unresectable non-small cell lung cancer. Lung Cancer. 2004;43(2):195-201. doi:10.1016/j.lungcan.2003.08.021

12. Santoro A, Tomino C, Prinzi G, et al. Tobacco smoking: risk to develop addiction, chronic obstructive pulmonary disease, and lung cancer. Recent Pat Anticancer Drug Discov. 2019;14(1):39-52. doi:10.2174/1574892814666190102122848

13. Tsukazan MTR, Vigo A, Silva VDD, Barrios CH, Rios JO, Pinto JAF. Lung cancer: changes in histology, gender, and age over the last 30 years in Brazil. J Bras Pneumol. 2017;43(5):363-367. doi:10.1590/s1806-37562016000000339

14. Jayia PK, Mishra PK, Shah RR, Panayiotou A, Yiu P, Luckraz H. Preoperative assessment of lung cancer patients: evaluating guideline compliance (re-audit). Asian Cardiovasc Thorac Ann. 2015;23 (3):299-301. doi:10.1177/0218492314552298

15. Detterbeck FC, Boffa DJ, Tanoue LT. The new lung cancer staging system. Chest. 2009;136(1):260-271. doi:10.1378/chest.08-0978

16. Mao W, Ma B, Huang X, et al. Which treatment is best for patients with AJCC stage IV bladder cancer? Int Urol Nephrol. 2019;51 (7):1145-1156. doi:10.1007/s11255-019-02105-5

17. Liu G, Xu M, Gao T, et al. Surgical compliance and outcomes in gastric cancer: a population-based cohort study. J Cancer. 2019;10 (4):779-788. doi:10.7150/jca.29073

18. Adesunkanmi AR, Lawal OO, Adelusola KA, Durosimi MA. The severity, outcome and challenges of breast cancer in Nigeria. Breast. 2006;15(3):399-409. doi:10.1016/j.breast.2005.06.008

19. Adham M, Stoker SD, Wildeman MA, et al. Current status of cancer care for young patients with nasopharyngeal carcinoma in Jakarta, Indonesia. PLoS One. 2014;9(7):e102353. doi:10.1371/journal. pone. 0102353

20. Mao W, Huang X, Kong M, Fan J, Geng J. More lymph node dissection improves survival in patients with newly diagnosed lymph node-positive penile cancer. Int Urol Nephrol. 2019;51 (4):641-654. doi:10.1007/s11255-019-02084-7
21. Filippetti M, Crucitti G, Andreetti C, et al. Experience of 10 years with the surgical treatment of lung cancer in elderly patients. Chir Ital. 2001;53(2):167-174.

22. Maze Y, Tenpaku H, Sato T. Assessment of surgery for patients older than 75 years of age with lung cancer. Kyobu Geka. 2006;59(2):89-92; discussion 92-84.

23. Cella DF, Orav EJ, Kornblith AB, et al. Socioeconomic-status and cancer survival. J Clin Oncol. 1991;9(8):1500-1509. doi:10.1200/ JCO.1991.9.8.1500

24. Munoz-Largacha JA, Steiling KA, Kathuria H, et al. Initial surgical experience following implementation of lung cancer screening at an urban safety net hospital. J Thorac Cardiovasc Surg. 2018;155 (6):2674-2679. doi:10.1016/j.jtcvs.2017.12.135

25. Salloum RG, Smith TJ, Jensen GA, Lafata JE. Factors associated with adherence to chemotherapy guidelines in patients with non-small cell lung cancer. Lung Cancer. 2012;75(2):255-260. doi:10.1016/j.lungcan.2011.07.005

26. Stessin AM, Sherr DL. Demographic disparities in patterns of care and survival outcomes for patients with resected gastric adenocarcinoma. Cancer Epidemiol Biomarkers. 2011;20 (2):223-233. doi:10.1158/1055-9965.EPI-10-0158

27. Cheville AL, Kollasch J, Vandenberg J, et al. A home-based exercise program to improve function, fatigue, and sleep quality in patients with Stage IV lung and colorectal cancer: a randomized controlled trial. J Pain Symptom Manage. 2013;45(5):811-821. doi:10.1016/j. jpainsymman.2012.05.006

28. Coats V, Maltais F, Simard S, et al. Feasibility and effectiveness of a home-based exercise training program before lung resection surgery. Can Respir J. 2013;20(2):E10-E16. doi:10.1155/2013/291059

29. Hinyard L, Wirth LS, Clancy JM, Schwartz T. The effect of marital status on breast cancer-related outcomes in women under 65: a SEER database analysis. Breast. 2017;32:13-17. doi:10.1016/j.breast. 2016.12.008

30. Thuret R, Sun M, Budaus L, et al. A population-based analysis of the effect of marital status on overall and cancer-specific mortality in patients with squamous cell carcinoma of the penis. Cancer Cause Control. 2013;24(1):71-79. doi:10.1007/s10552-012-0091-y

31. Jin JJ, Wang W, Dai FX, et al. Marital status and survival in patients with gastric cancer. Cancer Med. 2016;5(8):1821-1829. doi:10.1002/ cam4.758

32. El-Haddad B, Dong F, Kallail KJ, Hines RB, Ablah E. Association of marital status and colorectal cancer screening participation in the USA. Colorectal Dis. 2015;17(5):O108-O114. doi:10.1111/codi. 12926

33. Tantraworasin A, Taioli E, Liu B, Flores RM, Kaufman AJ. The influence of insurance type on stage at presentation, treatment, and survival between Asian American and non-Hispanic White lung cancer patients. Cancer Med. 2018;7(5):1612-1629. doi:10.1002/ cam4.1331

\section{Publish your work in this journal}

Cancer Management and Research is an international, peer-reviewed open access journal focusing on cancer research and the optimal use of preventative and integrated treatment interventions to achieve improved outcomes, enhanced survival and quality of life for the cancer patient.
The manuscript management system is completely online and includes a very quick and fair peer-review system, which is all easy to use. Visit http://www.dovepress.com/testimonials.php to read real quotes from published authors. 